\title{
Assessing Ability to Execute in Virtual Enterprises
}

\author{
Roelof J. van den Berg \\ Baan Development, The Netherlands \\ Em:rvdberg@baan.nl \\ Martin Tølle \\ Technical University of Denmark \\ Em: mat@ipt.dk
}

Keywords Virtual enterprise, ontological convergence, modelling, web assurance services

\begin{abstract}
The state of the art in ICT enables integration beyond the application per se, but this requires assessment and reconciliation of business processes. This paper describes this challenge to true co-operation in a virtual enterprise, focusing on industries for one-of-a-kind production. It explains why expressive modelling is important in the assessment process, by introducing the underlying mechanisms of inter-subjective maintenance of mental models. Finally, the paper discusses the future of assessment and auditing services in the context of business-to-business e-commerce.
\end{abstract}

\section{INTRODUCTION}

Most literature on e-commerce, including commerce in a business-tobusiness setting, concentrates on trading of standard products. Certainly, supporting the buying and selling of this type of products with advanced information technology can lead to significant gains in efficiency. But most business relationships go beyond mere trading. Their value is based on true co-operation, and synergy through mutual tuning of world views and processes. Prior to transactions in this context it is not automatically clear what each partner has to do nor if his business process execution matches with the contributions from others. Particularly in an arena centred around one-of-a-kind products e.g. power plants, ships or bridges resolving this ambiguity is no trivial endeavour. 
This paper highlights some aspects of assessment of ability to execute in virtual enterprises. It will start with a reflection on integration of information systems in section 2, focusing on the difference between deterministic and opportunistic integration. Section 3 and 4 are devoted to the main challenge of the latter type of integration. Section 5 will briefly discuss the context of the assessment, while section 6 contains the conclusions.

\section{EVOLUTION OF SYSTEM INTEGRATION}

Many predict that Internet will eventually enable one global so-called perfect market, where everybody can deal with everybody else on equal terms. This is based on the idea that price is all that matters. Certainly developments in trade of books, CD's or airplane flights support this. But in large segments of b-to-b commerce it pays to invest in differentiation and uniqueness through better product quality or logistical performance. Thus a consortium can essentially create a qualitative monopoly. In those areas of b-to-b commerce the trend is not so much towards one electronic market, but instead to electronic "balkanization".

In this situation several networks exist. The network is a co-operative alliance of competencies established to jointly exploit business opportunities. One enterprise, a group of enterprises or alternatively a large customer, who can be perceived as the business concept owner, initiates the creation of the network. Each network is relatively closed, allowing each member to exchange intimate business information with the others. The mutual trust between partners is the basis for much more sophisticated cooperation, up to the point that members seem to be part of one and the same "virtual" enterprise.

A virtual enterprise (VE) can be defined as a customer solutions delivery system created by a temporary and reconfigurable aggregation of core competencies. Note that in this view and unlike popular thought, members of successions of VEs always come from one and the same network. The network can be seen as a potential from which different VEs can be established in order to satisfy diverse customer demands. Although it comprises competencies from various partners, the VE appears to the customer as one, unified, and attuned enterprise. Hence its virtual nature. When the customer demand has been fulfilled, the virtual enterprise dissolves into its constituent parts to reassemble into other configurations (other VEs). Competencies and experiences gained in the virtual enterprise are transferred back to the network and its participants. See [4] for a more elaborate discussion on these issues. 
The developments concerning VEs mark a new era of integration of information systems. Many difficulties from earlier stages of integration of information systems have been addressed [5], but new challenges have appeared. We will further explain this evolution of enterprise integration with the layered framework, illustrated in figure 1. According to this framework satisfactory integration at a lower level is necessary before integration at a higher level can be achieved. We will briefly discuss each of the layers subsequently.

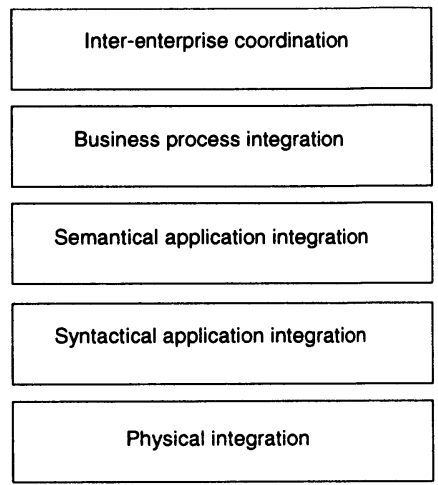

Figure 1 Framework for Enterprise Integration

\section{Standards for physical integration}

Naturally, physical integration is needed to facilitate co-operating applications and enterprises. Recently wireless integration has rapidly grown in importance. Relevant standards at this level of integration are WAP, MAP, and Ethernet.

\section{Syntactical standards for application integration}

This concerns the integration of application software systems at the level of "form". Standards in this area are Java, XML, Corba, DCOM.

\section{Semantic standards for application integration}

Semantic integration should result in application output which is meaningful to other applications. This type of integration abstracts from the technical details of software implementations. Examples of standards at this level are EDIFACT, STEP and BizTalk.

\section{Modelling languages for business process integration}

Virtual enterprises require a common understanding about the shared business processes. Modelling languages are needed to make these business processes explicit. Examples of standards at this level are IDEF, Petri nets, UML, ER, LOTOS, SDL, VDM, Z, B, •. Time is generally incorporated in these modelling languages, but distribution in space usually is not.

\section{Inter-enterprise co-ordination}


Dedicated guidelines at the level of inter-enterprise co-ordination are needed, e.g. for partner selection, certification or inter-enterprise best practice definition.

The framework in figure 1 is not specific to the era of "the new economy", but over the years the higher levels of the framework have become more relevant. Until recently integration typically concerned two given systems within two given units, often within one organization. The aim was to share specific data and applications for given purposes in fairly stabile business processes.

Without trying to marginalize that type of integration we could classify it as deterministic. Many aspects were known in advance, e.g. the business context of the integration, the players, their roles and their systems. The solution was dedicated to a specific case. Due to lack of standardization the emphasis in the integration effort was on the technical challenge of making the two information systems operate as one.

Currently most attention is devoted to a type of systems integration which is quite different, one based on much more standardized technology, enabling communication between information systems in enterprises without a priori acquaintance. The technical integration challenge for companies nowadays is to create "openness" to systems which are not known and owned by unknown partners, to enable co-operation in business functions which are not automatically streamlined across the VE.

Compared to the first type of integration, the second type is much less directed. It has to be more flexible to a wider variety of business applications. It is more opportunistic. ICT is used to release intellectual potential, through smart forms of co-operation. The two lower layers are becoming more and more standardized and a commodity, leaving more room to creatively exploit the benefits of open technologies. Thus the emphasis has shifted towards the three highest levels. In the next sections we will further discuss aspects of the fourth and fifth level respectively.

\section{THE CHALLENGE OF BUSINESS INTEGRATION}

Our view on business integration is based on the idea that the world around us is not just a given [3]. We influence it and, at a cognitive level, make it what it is. During our lives we create subjective realities, our worldviews. As a productive system our society is based on a separation of concerns, which breeds variety in world-views. A worker will be very much the product of his particular education (e.g. the ideas of a particular professor or "school"), professional culture (e.g. accountancy versus tax law) or organizational (sub) culture (e.g. Dow versus Shell versus BP or 
sales versus human resources or R\&D). We can say that specialization breeds ontological divergence: differentiation in world-views.

The variety in world-views is also reflected in our information systems. In a sense people try to freeze their world-view into the information systems they use. The way a department defines an entity e.g. "customer" or a business process e.g. "purchase order processing" depends on the precise way in which they operate, which in turn reflects their view on their role in the organization and its larger environment. This view is continuously maintained through the department's interaction with its environment. The main reason why large portions of systems development budgets are devoted to systems maintenance is the fact that world-views are not fixed.

The systems we employ become more and more open, but this does not mean their output automatically matches with the mental models of "foreign" users. To the contrary, open systems technology has only increased our possibilities to integrate our systems with those of others, which are based on increasingly different assumptions. The challenge of business integration has increased accordingly, but enterprises have to face it to stay competitive. Thus, especially in an era of open systems, mechanisms have to be available to bring world-views together, achieve ontological convergence and realize the benefits embedded in the state of the art of ICT.

\section{FUNCTIONAL CONFORMITY}

Concerning integration most literature on e-commerce concentrates on technical conformity: do the companies' respective systems comply with the same ICT-standards, so they can exchange data? We want to stress that in order to be competitive and really exploit the opportunities of enterprise integration another type of conformity should also be addressed: functional conformity. This addresses the question: does a common view exist on the inter-organizational activities and do business processes match (inputs/outputs required, processing sequence, lead times)?

It should be noted that a positive answer to this question does not mean that the ontological divergence itself should be denied and only standard solutions should be provided. As mentioned above clear reasons exist for the divergence in world-views. But in order to rapidly deploy integrations that truly support the operations in the VE it is necessary that the respective members make their views explicit. From there a solution can be set-up for their specific VE.

It is unrealistic to assume that each VE will be supported by a fully dedicated systems implementation. The temporary nature of the VE is one reason. Another is the fact that especially larger organizations will 
participate in more than one VE at the same time, with a desire to use the same ICT support. It is therefore required that they can support different approaches (for different business partners, regions, products etc.) with the same system simultaneously.

Current standard software solutions are able to accommodate for different approaches along a series of parameters, but they have great difficulty to support alternatives simultaneously within one implementation [2]. One reason is they are not enterprise "aware": the organizational scope of the functionality has not been modeled explicitly. Future systems will have to run in a powerful enterprise modelling environment, which includes an expressive dynamic model of the implemented enterprise systems themselves and of the ICT-infrastructure which is available: the model is the application.

Differentiated business processes have to be described in a form, understandable by humans and interpretable by software. It is especially this interpretation that should be enhanced, e.g. to allow different versions of software to semantically function similarly at different places in an enterprise. A necessary condition is that software can inspect at run time the configuration of versions, functions, processes, enterprise units, and data sets in its environment. On the other hand it should also be possible to access and activate the same enterprise software object through different business model instances.

\section{ASSESSMENT ISSUES: PRESENT AND FUTURE}

The human interpretation of the business models is an essential part of the assessment of ability to execute. Modelling techniques such as those for process modelling can be used to support this check for functional conformity. Current modelling techniques do not only enable enterprises to define their business processes at a generic "what do we do?" level, but also to clearly differentiate between process variants for different business partners, products, regions, seasons et cetera. Several business modelling tools make it possible to show which variants of business processes are active under specific circum- stances.

While the specification of the business processes has to be done inhouse, this does not apply to the assessment itself. After all, the ideal of VEs is driven by the strategic desire to concentrate on one's core business. From this perspective it is not sensible to have a large in-house assessment function.

Assessment should be left to specialized third parties. At this moment certified public accountants (CPAs) are most advanced in this area, 
especially those in North America [1]. In the past years they launched several web certification services for b-to-c commerce, but still have to launch one dedicated to b-to-b commerce. Other professionals, e.g. EDPauditors and even public notaries or banks could play a role in certifying enterprises which meet certain thresholds to participate in VEs.

Instead of making a definite choice for one group or the other, we raise the possibility to launch dedicated web assurance agencies, which combine expertise from several professions, e.g. accounting, EDP-auditing, law, payment systems, system integration, electronic marketing and cryptography. These agencies could perform an audit in an inter-disciplinary team. To support recognition of web assurance services, it is important to establish a standardized way of working as much as possible. Among the CPAs mentioned above a tendency exists to develop a firm specific web assurance provision with corresponding seal. A "jungle" of unclear web assurance services and corresponding certifications only leads to confusion in the target market.

More specifically the assessment should be organized as a mix of a public and private function. Public functions are strictly regulated by law to secure standardization of execution and to provide outsiders with a solid basis for interpretation of the results. An example of a public function of CPA's is the annual audit of the financial statements of a company. The generic parts of assessments, resulting in conclusions that go beyond the lifetime of one VE, can be organized as a public function: an enterprise is evaluated in isolation vis-a-vis generally accepted standards. Results of such public assessments could be used to recruit enterprises as part of a network.

Unlike public assessments private ones are reported to the client only. For this reason the precise organization of such an audit is much more left to the discretion of the individual auditing firm. In a web assurance audit a private (part of an) assessment will deal with the specific requirements of the VE that is being set up. The precise nature of the audit will be tuned to the requirements of the network, which requests it, and the exact work that has to be executed in the VE. In a private assessment the enterprise is evaluated reciprocally against the needs of others in the VE. It is not unlikely that application service providers will eventually take the web assurance services under their wing.

\section{CONCLUSIONS}

Current rhetoric on e-commerce tends to depreciate the complexity of requirements for true co-operation in e-business as opposed to merely 
trading. Developments in open systems technology certainly have pushed the state of the art in technical integration of systems, but they do not automatically support additional business integration which is required to establish VEs.

This last type of integration cannot be achieved without reconciliation of the world-views that underlie the systems in the VE. Explicit modelling of the assumptions behind each of the partners' systems functionality is needed to support this ontological convergence. Modelling can also help to differentiate functionality of one and the same system along various dimensions. This requires executable modelling environments. The model becomes the application. Eventually dedicated web assurance service providers will assess a partner's ability to execute in a VE. The generic assessment should be organized as a public function similar to a CPA audit of a firm's annual accounts or ISO certification. The subsequent more specific assessment per VE is best organized as a dedicated private function. In the future it is likely that application service providers will cover the assessment function as a natural outcome of their mission to provide the ICT backbones of VEs.

\section{ACKNOWLEDGEMENT}

This paper benefited from the critical review and valuable remarks from Jens Dahl-Pedersen at the Technical University of Denmark and Arian Zwegers at Baan Development.

\section{REFERENCES}

[1] Berg, R.J. van den and Lieshout, van, J.M., "Eliminating Hurdles to Trust in

E-commerce", in Global Production Management, K. Mertens, O. Krause and B. Schallock (eds.), Kluwer, 1999, pp. 522-529.

[2] Berg, R.J. van den, and Zwegers, A.J.R.,"'Decoupling Functionality to Facilitate

Controlled Growth", in Studies in Informatics and Control, Vol. 6, No. 1, March 1997, pp. 57-64.

[3] Berger, Peter L. and Luckmann, Thomas, The Social Construction of Reality, Penguin University Books, Middlesex, 1971.

[4] Tølle, M., Vesterager, J., Dahl-Pedersen, J., "A Methodology for Virtual Enterprise Management - Results from IMS 95001/Esprit 26509 Globeman 21 project”, Proceedings of the $6^{\text {th }}$ International Conference on Concurrent Enterprising, Toulouse, 28-30 June 2000, pp. 119-127.

[5] Zwegers, A.J.R., On Systems Architecting, Ph.D. thesis, Eindhoven University of Technology, 1998. 